\title{
Skin disorders in Parkinson's disease: potential biomarkers and risk factors
}

\section{Astrid-Helene Ravn Jacob P Thyssen \\ Alexander Egeberg}

Department of Dermatology and Allergy, Herlev and Gentofte Hospital, University of Copenhagen, Hellerup, Denmark
Correspondence: Alexander Egeberg Department of Dermatology and Allergy, Herlev and Gentofte Hospital, University of Copenhagen, 2900 Hellerup, Denmark Tel +45 242I 542 I

Email alexander.egeberg@gmail.com
This article was published in the following Dove Press journal:

Clinical, Cosmetic and Investigational Dermatology

9 March 2017

Number of times this article has been viewed

\begin{abstract}
Parkinson's disease (PD) is one of the most common neurodegenerative disorders, characterized by a symptom triad comprising resting tremor, rigidity, and akinesia. In addition, non-motor symptoms of PD are well recognized and often precede the overt motor manifestations. Cutaneous manifestations as markers of PD have long been discussed, and cumulative evidence shows an increased prevalence of certain dermatological disorders in PD. Seborrheic dermatitis is considered to occur as a premotor feature of PD referable to dysregulation of the autonomic nervous system. Also, an increased risk of melanoma has been observed in PD. Light hair color is a known risk factor for melanoma, and interestingly the risk of PD is found to be significantly higher in individuals with light hair color and particularly with red hair. Furthermore, several studies have reported a high prevalence of PD in patients with bullous pemphigoid. Moreover, a 2-fold increase in risk of new-onset PD has been observed in patients with rosacea. Besides the association between PD and various dermatological disorders, the skin may be useful in the diagnosis of PD. Early PD pathology is found not only in the brain but also in extra-neuronal tissues. Thus, the protein $\alpha$-synuclein, which is genetically associated with PD, is present not only in the CNS but also in the skin. Hence, higher values of $\alpha$-synuclein have been observed in the skin of patients with PD. Furthermore, an increased risk of PD has been found in the Cys/ Cys genotype, which is associated with red hair color. In this review, we summarize the current evidence of the association between PD and dermatological disorders, the cutaneous adverse effects of neurological medications, and describe the potential of skin protein expression and biomarkers in identification of risk and diagnosis of PD.
\end{abstract}

Keywords: Parkinson's disease, melanoma, rosacea, seborrheic dermatitis, bullous pemphigoid

\section{Introduction}

Parkinson's disease (PD) is one of the most common neurodegenerative disorders, ${ }^{1}$ affecting $\sim 0.3 \%$ of the general population in industrialized countries. ${ }^{1}$ Although the pathogenesis of PD is not yet fully understood, the selective loss of dopaminergic neurons is believed to play a crucial role in disease onset and progression. Explanatory theories include mitochondrial dysfunction, oxidative stress, and protein mishandling. ${ }^{1}$ Moreover, although most cases occur sporadically, several genetic mutations, for example, in the Parkin (PARK2) and PINK1 (PARKO) genes have also been associated with the disease. ${ }^{2}$

$\mathrm{PD}$ is characterized by a symptom triad comprising resting tremor, rigidity, and akinesia. In addition, non-motor symptoms of PD are well recognized and often precede the overt motor manifestations. ${ }^{3}$ The non-motor symptoms of PD include sleep disorders, constipation, bladder problems, excessive saliva, depression, apathy, fear and anxiety, and cutaneous problems. ${ }^{3}$ 
The latter have been discussed since the beginning of the 20th century, and cumulative evidence suggests a causal association between the neurological disturbance in PD and associated cutaneous manifestations. ${ }^{4}$ Despite the idea that certain dermatological disorders are frequent non-motor symptoms of $\mathrm{PD}$, and potentially add to the impaired quality of life in these patients, these often go unnoticed by the treating physicians. ${ }^{4}$

Attention should be paid to PD-associated skin disorders as they affect the psychosocial well-being of the patients. It is, therefore, important to correctly diagnose and treat the disorders to improve the patients' quality of life.

In this review, we summarize the current evidence of dermatological disorders in patients with $\mathrm{PD}$, outline selected cutaneous adverse effects of anti-PD medications, and discuss how objective skin changes can serve as a diagnostic tool in PD.

The PubMed and Medline databases were searched (September 2016) to identify studies investigating PD-associated skin disorders. The references of the eligible literature were assessed to identify additional relevant studies.

\section{Skin conditions in PD Seborrheic dermatitis}

Seborrheic dermatitis is a common chronic inflammatory skin disorder, affecting $\sim 1 \%-3 \%$ of the general population (Figure 1). ${ }^{5}$ It is primarily located to sebum-rich areas, such as the scalp, face, hairline, eyebrow, glabella, nasolabial folds, ears, upper chest, and in skin folds under arms and on legs. The incidence of seborrheic dermatitis peaks in certain age groups, that is, infants up to 3 months old, during puberty, and in adulthood. ${ }^{6}$ In all age groups, an incidence of $3.0 \%$ is found in men vs. $2.6 \%$ in women, thus, suggesting a pathogenic role of certain sex hormones. ${ }^{7}$

The increased production of host sebum as well as the presence and increased reproduction of Malassezia yeasts are

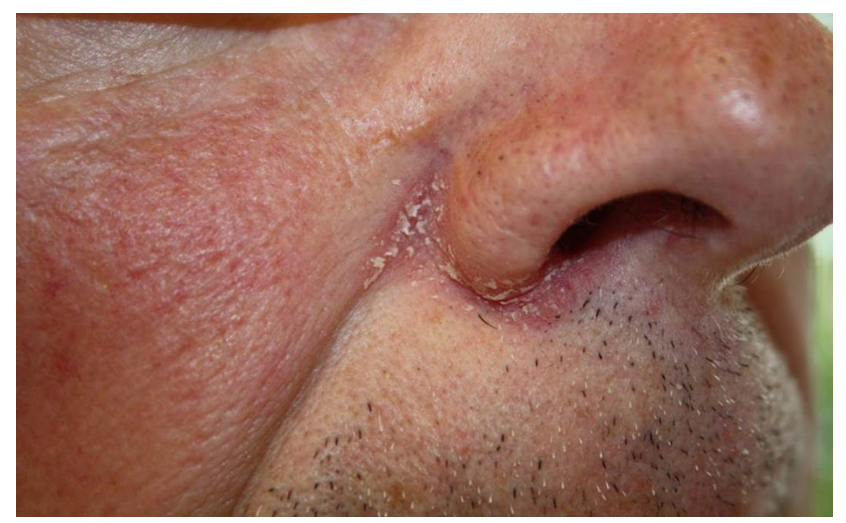

Figure I Seborrheic dermatitis. the two major factors believed to contribute to the development of SD. ${ }^{4,8}$ Seborrhea has been associated with PD, with a prevalence as high as 52\%-59\%. ${ }^{8}$ In 1927, Krestin reported seborrheic facies as a cutaneous manifestation of parkinsonism, particularly in post-encephalic parkinsonism, ${ }^{9}$ and described the skin as shiny, greasy, and with characteristic scarring acne. In a retrospective study on the association between seborrheic dermatitis and PD, Tanner et al proposed that seborrheic dermatitis might represent a premotor feature of PD, ascribable to autonomic nervous system dysregulation, and suggested that seborrheic dermatitis could serve as an early disease marker of PD. ${ }^{10}$ Appropriate anti-fungal treatment, such as ketoconazole can be useful for PD patients, as such agents may reduce Malassezia growth and enzyme production. Anti-inflammatory agents in the form of topical steroids or topical calcineurin inhibitors can also be used in the treatment, thus improving patient's well-being and quality of life.

\section{Melanoma}

Melanoma is a skin cancer arising from epidermal melanocytes (Figure 2). Risk factors for melanoma comprise a combination of constitutional predisposition (fair skin, blond or red hair, freckles, family history of melanoma, high number of nevi, and increasing age) and exposure to ultraviolet radiation. ${ }^{11,12}$

The exact risk of melanoma in PD remains unclear. A connection was first suspected following a number of case reports, suggesting that levodopa therapy might increase the risk of melanoma. ${ }^{13}$ However, this finding was later questioned after reviews of these case reports concluded that the link was coincidental rather than causal. ${ }^{14-16}$ Nevertheless,

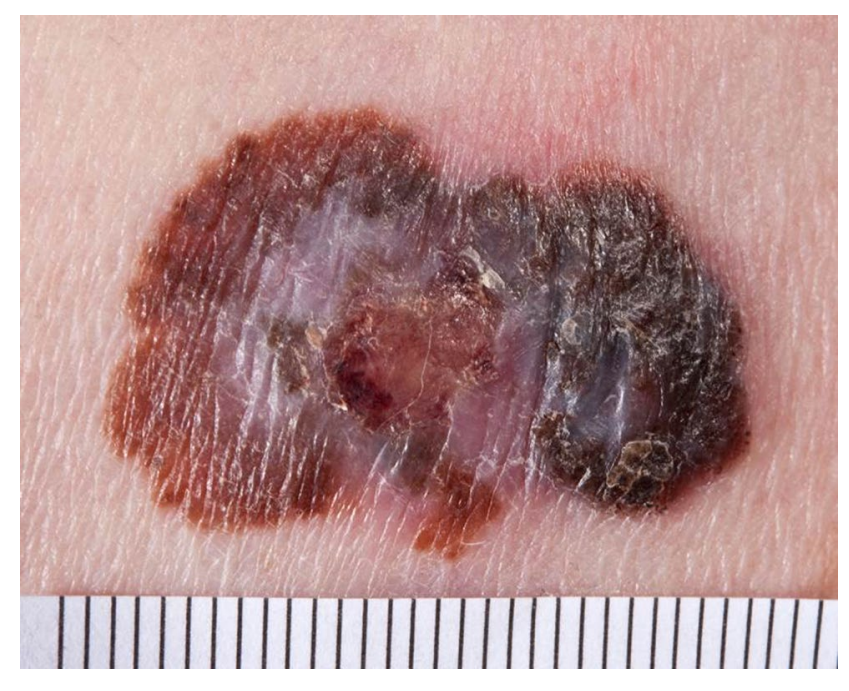

Figure 2 Malignant melanoma. 
studies in favor of a possible association between PD and melanoma have been published. A large Danish study of $>14,000$ patients with $\mathrm{PD}$ concluded that an increased rate of melanoma was restricted to those with idiopathic PD and unrelated to levodopa treatment. ${ }^{17}$ Similarly, a prospective study including 2106 patients from North America found a 2-fold higher melanoma prevalence in patients with PD compared with the general population, ${ }^{18}$ yet a large metaanalysis only found a moderate association between the two conditions. ${ }^{19}$

The explanation for a potential link between melanoma and PD is unclear, but various hypotheses have been put forward. $\alpha$-Synuclein, an enzyme that inhibits tyrosine hydroxylase, is involved in the melanin synthesis in both melanoma and dopaminergic neuronal cells in PD and could be one plausible explanation..$^{20}$ Moreover, while light hair color is a risk factor for melanoma, a prospective study including 132,302 health care professionals, ${ }^{21}$ found that risk of PD increased significantly with decreasing darkness of hair color. Indeed, red-haired individuals had an $\sim 2$-fold higher PD risk compared with black-haired individuals. Furthermore, an association between hair color and earlyonset PD (<70 years) was also observed, whereas hair color was not significantly associated with risk of PD onset after the age of 70 years. Although the exact cause remains to be elucidated, differences in pigment metabolism may explain the observed association of PD with lighter hair colors. ${ }^{21}$ Consequently, physicians could advise patients with PD, in particular those with lighter skin tones, on the possible augmented melanoma risk and encourage them to remain vigilant about any new, or changing pigmented skin lesions. Furthermore, emphasis on the importance of sun protection, including limiting exposure and using high-factor sun blocking agents, could be valuable. ${ }^{22}$

\section{Bullous pemphigoid}

Bullous pemphigoid (BP) is a bullous autoimmune skin disease, affecting elderly people with a median age of 80 years (Figure 3). ${ }^{23,24}$ The condition is characterized by pruritus and tense cutaneous bullae on erythematous skin and is associated with significantly increased mortality rates compared with the general population. ${ }^{24,25}$ The pathogenesis of BP includes the development of autoantibodies against skin antigens, ${ }^{24}$ but the pathogenic link with PD remains poorly understood. Several retrospective studies have reported associations between previous neurological diseases, including PD, and the later development of BP. In a study by Cordel et al, ${ }^{26}$ PD was observed in $32(9 \%)$ of 341 patients with BP. The

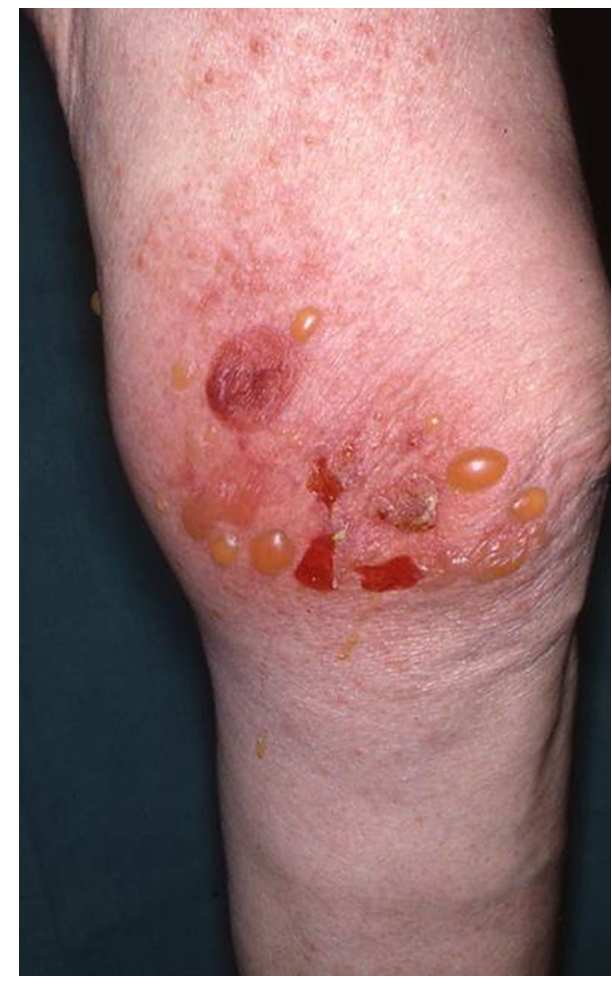

Figure 3 Bullous pemphigoid.

association was also found in a population-based case-control study including 868 patients with BP and 3453 controls where PD was present in 26 cases (3\%) and in 36 controls $(1 \%),{ }^{24}$ and similar findings were reported in a retrospective study from Olmsted County, MN. ${ }^{25}$ Along this line, a Spanish case-control study, including 56 patients with BP and 112 control subjects, reported a PD prevalence of $17.9 \%$ and $3.6 \%$ among BP patients and controls, respectively. ${ }^{27}$ Finally, a multi-center case-control study evaluating the risk factors for BP in elderly people found PD present in 14.3\% of BP cases compared with only $5.8 \%$ control subjects. ${ }^{28}$ As BP increases mortality and affects the psychosocial well-being, it is important to correctly diagnose and treat the disease to improve the patients' quality of life.

\section{Rosacea}

Rosacea, a common facial skin dermatosis, is characterized by the features of neurogenic inflammation, such as transient or persistent facial erythema, edema, burning pain, immune infiltration, telangiectasia, papules, and pustules, and has an estimated prevalence ranging from $1 \%$ to $20 \%$ (Figure 4 ). ${ }^{29,30}$ Emerging evidence suggests a possible association between rosacea and $\mathrm{PD} .{ }^{31-33}$ In a study by Fischer et al, ${ }^{31}$ non-invasive bioengineering methods (sebumetry, corneometry, $\mathrm{pH}$ involving 70 patients with PD, rosacea was present in $18.6 \%$, and flushing symptoms were found in $31.9 \%$ of patients. The 


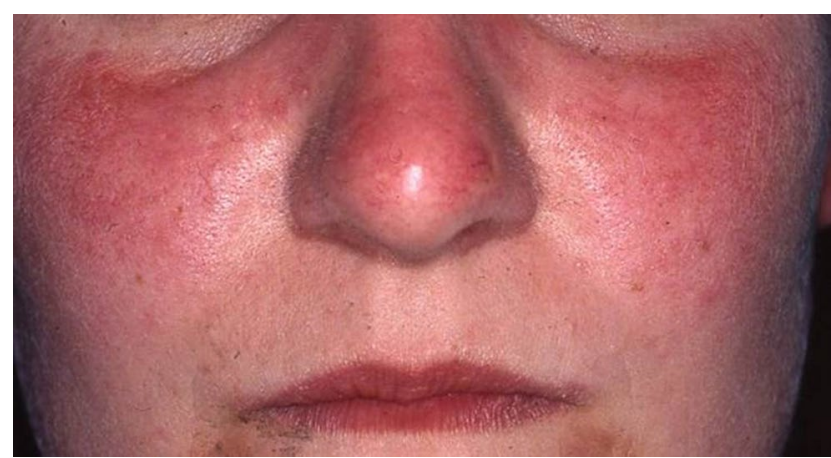

Figure 4 Rosacea.

overlap between the facial flushing occurring in PD due to autonomic dysregulation and the facial flushing experienced in patients with rosacea was noted as a complicating factor in the diagnosis of concurrent rosacea and PD. Importantly, in a nationwide Danish cohort study of $>4,000,000$ individuals, an $\sim 2$-fold increase in risk of new-onset PD was observed in patients with rosacea. ${ }^{32}$ This finding was very recently replicated in a US cohort using electronic medical record linkage of 803,005 individuals, including 17,682 patients with rosacea. ${ }^{33}$

Although the pathogenic link between rosacea and PD has not yet been fully determined, shared pathogenic mechanisms involving elevated matrix metalloproteinase activity have been suggested. ${ }^{32}$ In addition, studies have observed a decreased density of cutaneous nerve fibers in the early and late stages of PD. ${ }^{34}$ This dysfunction of sensory nerve fibers contributes to the release of a sectional immune reaction, following the activation of the immune response. This immune destabilization is believed to result in occurrence of opportunistic skin disorders in vulnerable areas of the skin. ${ }^{35}$ As non-motor symptoms often precede the motor symptoms in PD, it is possible that PD may foreword the clinical manifestation of rosacea. Therefore, rosacea may constitute an independent risk factor for PD and vice versa. ${ }^{35}$ The gastrointestinal system has also been proposed as a link between the two conditions. As the entire gastrointestinal tract (GIT) is affected in patients with PD, increasing evidence indicates that the GIT indeed might be the site of disease initiation. ${ }^{36}$ Moreover, persistent infection with small intestine bacterial overgrowth and Helicobacter pylori may contribute to the pathogenesis. ${ }^{37}$ Although speculative, the recent observations could suggest that cutaneous rosacea features may be an early disease marker of PD in certain patients.

\section{Cutaneous adverse effects of neurological medications}

While allergic cutaneous reactions to dopaminergic drugs are very rare, few cases of skin rashes have been reported following the use of Sinemet ${ }^{\circledR}$ (carbidopa/levodopa) compounded with yellow dye. As rashes were not reported with other tablet sizes of this drug containing blue dye, the yellow dye used in that specific preparation was suspected as the offending compound. ${ }^{4,38}$ The cases reported maculo-papular rashes on the trunk and arms, which resolved following discontinuation of carbidopa/levodopa and reoccurred when the drug was reintroduced.

Symmetrel $^{\circledR}$ (Amantaine), a primary amine originally used as an antiviral chemotherapeutic, was later found efficacious as an anti-Parkinson agent. The drugs have been known to cause a mottled reticulated vascular pattern, termed livedo reticularis (LR), which is predominantly localized on the lower extremities. ${ }^{39}$ The exact cause of the development of LR following or during treatment with amantadine remains unclear, but may be due to interruption of the peripheral blood redistribution, particularly in the dermal arteries and peripheral vasospasms which lead to LR. Ergoline, a chemical compound used in certain dopamine agonists have been associated with erythromelalgia, a rare neurovascular skin disorder that usually affects the palms and soles of the hands and feet. It is characterized by episodes of erythema, swelling, and painful burning sensation. However, these days, ergoline dopamine agonists are rarely used for PD, and no cases of erythromelalgia have been reported with the use of the non-ergoline dopamine agonists. ${ }^{4}$

As various cutaneous effects may occur following the use of anti-PD agents, thorough patient information and regular monitoring by treating physicians is advised. Discontinuation of treatment can cause motor symptoms such as tremor and dyskinesia, but continued therapy following cutaneous reactions may potentially worsen the physical and mental conditions of patients with PD. Consequently, a personalized treatment approach is, therefore, recommended.

\section{Potential of skin protein expression in PD}

Beyond PD's association with various dermatological disorders, the skin is also likely to serve as a helpful tool in the diagnosis of PD. $\alpha$-Synuclein, a presynaptic neuronal protein and the main protein component of Lewy bodies, is genetically linked with PD. As emerging evidence has shown, $\alpha$-synuclein is not only found in the central nervous system but also found in the peripheral autonomic nervous system. ${ }^{20}$ Potential biomarkers allowing for a precise diagnosis of synucleinopathies have been investigated in body fluids and peripheral tissues, such as salivatory glands, the peripheral nervous system, and the skin. However, no 
widely accepted diagnostic marker presently exists. ${ }^{40-42}$ In one study, the presence of $\alpha$-synuclein was analyzed in the skin derived from punch biopsies of 17 healthy individuals and an identical number patients with PD. ${ }^{43}$ The study found that $\alpha$-synuclein values were significantly higher in the PD group compared with controls. Furthermore, Ikemura et al, prospectively examined skin samples from the abdominal wall and upper limbs of 279 patients of whom 85 patients displayed Lewy Body pathology. ${ }^{44}$ The authors found dermal $\alpha$-synuclein immunoreactivity in $23.5 \%$ of patients with Lewy Body pathology. No reactivity was observed in patients without Lewy Body pathology. Moreover, Miki et al examined dermal and subcutaneous tissue samples from the chest wall and lower limb of 20 patients with PD. ${ }^{45}$ Interestingly, while abnormal $\alpha$-synuclein accumulation was observed in the dermis of the chest wall, findings from the lower limbs were less consistent.

\section{Implications for biomarkers in the prediction and early diagnosis of Parkinson's disease}

Cumulative cellular damage in patients with PD can be found in skin fibroblasts, and the PINK1 and Parkin genes are expressed at relevant levels in human fibroblasts. ${ }^{2}$ Moreover, fibroblasts from patients with idiopathic PD show a clear and distinctive mRNA expression pattern of key genes in neurodegeneration, ${ }^{2}$ and increased risk of $\mathrm{PD}$ is found in specific genotypes. For example, a case-control study including 272 cases and 1185 controls found that participants with the Cys/ Cys genotype had a higher risk of PD. ${ }^{21}$ This specific genotype is associated with red hair, thus suggesting a potential role of pigmentation in PD. ${ }^{21}$

Conclusively, as early pathology is not only found in the brain but possibly also in extra-neuronal tissues such as the skin, it is tempting to speculate that skin biopsies may be used for PD diagnosis in the future. ${ }^{2}$ In addition, origins of skin-derived precursors may serve an alternative source of stem cells to embryonic stem cells for transplantation therapy for PD. ${ }^{46}$

\section{Conclusion and future directions}

In conclusion, patients with PD may suffer from a variety of concurrent or preceding skin disorders. While seborrheic dermatitis is considered to occur as a premotor symptom in PD, rosacea may constitute a risk factor, or an early sign of PD development. Furthermore, strong associations between PD and the later development of BP have also been found, and a possible link between PD and melanoma has been observed. As increased risk of PD and melanoma has been found in individuals with light hair color, and particularly red hair, treating physicians should show these individuals special awareness and emphasize the importance of sun protection, including limiting exposure and using high-factor sun blocking agents.

Analyses of $\alpha$-synuclein in skin biopsies show that pathophysiological examinations of neurodegenerative disorders, such as PD, may be successful through investigation of the skin. Therefore, skin biopsies may in the future be used in the diagnosis of PD. Yet, to better understand the association between PD and specific dermatological diseases in general, and BP in particular, further studies are required.

As skin disorders represent an important aspect of life with PD, awareness and correct treatment of these are important in order to improve patients' everyday life.

\section{Disclosure}

Dr. Egeberg has received research funding and/or consultancy honoraria from Pfizer and Eli Lilly, and honoraria as consultant and/or speaker from Pfizer, Eli Lilly, Novartis, Galderma, and Janssen Pharmaceuticals. Dr. Thyssen is supported by an unrestricted grant from the Lundbeck Foundation and has received speaker honoraria from Galderma and MEDA. The authors report no other conflicts of interest in this work.

\section{References}

1. de Lau LML, Breteler MMB. Epidemiology of Parkinson's disease. Lancet Neurol. 2006;5(6):525-535.

2. Auburger G, Klinkenberg M, Drost J, et al. Primary skin fibroblasts as a model of Parkinson's disease. Mol Neurobiol. 2012;46(1):20-27.

3. Chaudhuri KR, Healy DG, Schapira AH; National Institute for Clinical Excellence. Non-motor symptoms of Parkinson's disease: diagnosis and management. Lancet Neurol. 2006;5(3):235-245.

4. Gregory R, Miller S. Parkinson's disease and the skin. Pract Neurol. 2015;15(4):246-249.

5. Gupta AK, Madzia SE, Batra R. Etiology and management of seborrheic dermatitis. Dermatology. 2004;208(2):89-93.

6. Dessinioti C, Katsambas A. Seborrheic dermatitis: etiology, risk factors, and treatments: facts and controversies. Clin Dermatol. 2013;31(4):343-351.

7. Naldi L, Rebora A. Clinical practice. Seborrheic dermatitis. $N$ Engl J Med. 2009;360(4):387-396.

8. Arsic Arsenijevic VS, Milobratovic D, Barac AM, Vekic B, Marinkovic J, Kostic VS. A laboratory-based study on patients with Parkinson's disease and seborrheic dermatitis: the presence and density of Malassezia yeasts, their different species and enzymes production. BMC Dermatol. 2014;14:5.

9. Krestin D. The seborrhoeic facies as a manifestation of post-encephalitic Parkinsonism and allied disorders. QJM. 1927;21(81):177-186.

10. Tanner C, Albers K, Goldman S, et al. Seborrheic Dermatitis and Risk of Future Parkinson's Disease. Neurology. 2012;78(1):2012.

11. Gilchrest BA, Eller MS, Geller AC, Yaar M. The pathogenesis of melanoma induced by ultraviolet radiation. $N$ Engl J Med. 1999;340(17): 1341-1348. 
12. Diepgen TL, Mahler V. The epidemiology of skin cancer. Br J Dermatol. 2002;146 (Suppl 61):1-6.

13. Skibba JL, Pinckley J, Gilbert EF, Johnson RO. Multiple primary melanoma following administration of levodopa. Arch Pathol. 1972;93(6): $556-561$.

14. Fiala KH, Whetteckey J, Manyam B V. Malignant melanoma and levodopa in Parkinson's disease: causality or coincidence? Parkinsonism Relat Disord. 2003;9(6):321-327.

15. Pfutzner W, Przybilla B. Malignant melanoma and levodopa: is there a relationship? Two new cases and a review of the literature. $J$ Am Acad Dermatol. 1997;37(2 Pt 2):332-336.

16. Siple JF, Schneider DC, Wanlass WA, Rosenblatt BK. Levodopa therapy and the risk of malignant melanoma. Ann Pharmacother. 2000; 34(3):382-385.

17. Olsen JH, Tangerud K, Wermuth L, Frederiksen K, Friis S. Treatment with levodopa and risk for malignant melanoma. Mov Disord. 2007; 22(9):1252-1257.

18. Bertoni JM, Arlette JP, Fernandez HH, et al. Increased melanoma risk in Parkinson disease: a prospective clinicopathological study. Arch Neurol. 2010;67(3):347-352.

19. Liu R, Gao X, Lu Y, Chen H. Meta-analysis of the relationship between Parkinson disease and melanoma. Neurology. 2011;76(23): 2002-2009.

20. Pan T, Zhu J, Hwu W-J, Jankovic J. The role of alpha-synuclein in melanin synthesis in melanoma and dopaminergic neuronal cells. PLoS One. 2012;7(9):e45183.

21. Gao X, Simon KC, Han J, Schwarzschild MA, Ascherio A. Genetic determinants of hair color and Parkinson's disease risk. Ann Neurol. 2009;65(1):76-82.

22. Nikolaou V, Stratigos AJ. Emerging trends in the epidemiology of melanoma. Br J Dermatol. 2014;170(1):11-19.

23. Stinco G, Codutti R, Scarbolo M, Valent F, Patrone P. A retrospective epidemiological study on the association of bullous pemphigoid and neurological diseases. Acta Derm Venereol. 2005;85(2):136-139.

24. Langan SM, Groves RW, West J. The relationship between neurological disease and bullous pemphigoid: a population-based case-control study. J Invest Dermatol. 2011;131(3):631-636.

25. Brick KE, Weaver CH, Savica R, et al. A population-based study of the association between bullous pemphigoid and neurologic disorders. $J$ Am Acad Dermatol. 2014;71(6):1191-1197.

26. Cordel N, Chosidow O, Hellot M-F, et al. Neurological disorders in patients with bullous pemphigoid. Dermatology. 2007;215(3): 187-191.

27. Casas-de-la-Asuncion E, Ruano-Ruiz J, Rodriguez-Martin AM, Velez Garcia-Nieto A, Moreno-Gimenez JC. Association between bullous pemphigoid and neurologic diseases: a case-control study. Actas Dermosifiliogr. 2014;105(9):860-865.
28. Bastuji-Garin S, Joly P, Lemordant P, et al. Risk factors for bullous pemphigoid in the elderly: a prospective case-control study. $J$ Invest Dermatol. 2011;131(3):637-643.

29. Tan J, Berg M. Rosacea: current state of epidemiology. J Am Acad Dermatol. 2013;69(6 Suppl 1):S27-S35.

30. Schwab VD, Sulk M, Seeliger S, et al. Neurovascular and neuroimmune aspects in the pathophysiology of rosacea. J Investig dermatology Symp Proc. 2011;15(1):53-62.

31. Fischer M, Gemende I, Marsch WC, FischerPA. Skin function and skin disorders in Parkinson's disease. J Neural Transm. 2001;108(2): 205-213.

32. Egeberg A, Hansen PR, Gislason GH, Thyssen JP. Exploring the association between rosacea and Parkinson disease: a Danish nationwide cohort study. JAMA Neurol. 2016;73(5):529-534.

33. Lyon S, Majewski S, Guido N, et al. LB766 Parkinson's disease association with rosacea: a large, single center, retrospective study. J Invest Dermatol. 2016;136(8):B3.

34. Schneider SA, Boettner M, Alexoudi A, Zorenkov D, Deuschl G, Wedel T. Can we use peripheral tissue biopsies to diagnose Parkinson's disease? A review of the literature. Eur J Neurol. 2016;23(2):247-261.

35. Alexoudi A, Alexoudi I, Gatzonis S. Association between rosacea and Parkinson disease. JAMA Neurol. 2016;73(9):1159.

36. Alexoudi A, Alexoudi I, Gatzonis S. Association between rosacea and gastrointestinal disorders. Br J Dermatol. 2016;175(6):1405.

37. Fasano A, Visanji NP, Liu LWC, Lang AE, Pfeiffer RF. Gastrointestinal dysfunction in Parkinson's disease. Lancet Neurol. 2015;14(6):625-639.

38. Chou KL, Stacy MA. Skin rash associated with Sinemet does not equal levodopa allergy. Neurology. 2007;68(13):1078-1079.

39. Rana AQ, Masroor MS. Patient perception of Levido reticularis due to amantadine. Int J Neurosci. 2012;122(7):363-366.

40. Ludolph AC, Kassubek J, Landwehrmeyer BG, et al. Tauopathies with parkinsonism: clinical spectrum, neuropathologic basis, biological markers, and treatment options. Eur J Neurol. 2009;16(3):297-309.

41. Stamelou M, Hoeglinger GU. Atypical parkinsonism: an update. Curr Opin Neurol. 2013;26(4):401.i-405.i.

42. Lindow KB, Warren C. Understanding rosacea - a guide to facilitating care. Am J Nurs. 2001;101(10):44-51.

43. Rodriguez-Leyva I, Chi-Ahumada EG, Carrizales J, et al. Parkinson disease and progressive supranuclear palsy: protein expression in skin. Ann Clin Transl Neurol. 2016;3(3):191-199.

44. Ikemura M, Saito Y, Sengoku R, et al. Lewy body pathology involves cutaneous nerves. J Neuropathol Exp Neurol. 2008;67(10):945-953.

45. Miki Y, Tomiyama M, Ueno T, et al. Clinical availability of skin biopsy in the diagnosis of Parkinson's disease. Neurosci Lett. 2010;469(3):357-359.

46. Lu X, Tao Y, Li L. Prospective use of skin-derived precursors in neural regeneration. Chin Med J (Engl). 2012;125(24):4488-4496.
Clinical, Cosmetic and Investigational Dermatology

\section{Publish your work in this journal}

Clinical, Cosmetic and Investigational Dermatology is an international, peer-reviewed, open access, online journal that focuses on the latest clinical and experimental research in all aspects of skin disease and cosmetic interventions. This journal is included on PubMed. The manuscript management system is completely online

\section{Dovepress}

and includes a very quick and fair peer-review system, which is all easy to use. Visit http://www.dovepress.com/testimonials.php to read real quotes from published authors 